\title{
A Study on the Mediating Effect of Self-esteem in the Relationship between Aging Anxiety and Depression
}

\author{
Ok-Geun $\mathrm{Choi}^{1}$, Yun-Jeong Kim ${ }^{2}$ \\ ${ }^{1}$ Doctor Course, Department of Life Long Education, Hanseo University, Republic of Korea, \\ cokchunga@hanmail.net \\ ${ }^{2}$ Professor, Department of Health, Counselling and Welfare, Hanseo University, Republic of Korea, \\ twoyun21@hanmail.net
}

Corresponding author: Yun-Jeong Kim

\begin{abstract}
This study aims to understand the mediating effect of self-esteem in the effects of aging anxiety on depression in the middle-aged and elderly generations living in local communities. To achieve this purpose, a survey was conducted in December 2019 among 200 middle-aged people and 300 elderly residing in Seoul, Gyeonggi, and Seosan, Chungnam with the cooperation of the institutions such as the local lifelong learning centers, general social welfare centers, and volunteer centers, and the help of people in charge. In the case of the middle-aged, 200 copies were finally collected and used for analysis. In the case of the elderly, additional help was received from the elderly welfare centers. In general, 286 copies were used for analysis, excluding 14 copies of the questionnaire with no response. For the analysis, IBM SPSS 21.0 and SPSS PROCESS macro model No. 4 were used. The main research results are summarized as follows. First, it was found that aging anxiety and depression were higher in the elderly, and self-esteem was higher in the middle-aged. Second, as a result of verifying the mediating effect of self-esteem in the relationship between aging anxiety and depression, the higher the aging anxiety in both the middle-aged and elderly generations, the higher the feeling of depression, but the effect of aging anxiety on depression decreased when self-esteem was added. In other words, the mediating effect of self-esteem was verified in the relationship between aging anxiety and depression. These results are meaningful in that it revealed that the aging anxiety of the middle-aged and elderly generations causes depression, but if self-esteem is high, this influence could be weakened. Therefore, it is suggested a self-esteem improvement program for the middle-aged and elderly generations.
\end{abstract}

Keywords: Aging anxiety, Depression, Self-esteem, Mediating Effect, SPSS Macro Process Model 4

\section{Introduction}

Currently, the proportion of the population aged 65 and over in Korea is $15.7 \%$, and the life expectancy is 82.7 years[1], and the increase in the proportion of the elderly population has a great impact on society, which also has a great effect on quality. Humans live by experiencing aging from the moment they are born into the world. From infants to adolescents, they are not conscious of aging, but when they get out of the youth period, they become worried and anxious about aging[2]. Aging anxiety refers to anxiety and fear of changes, incompetence, and death experienced in the process of aging[3]. In the modern society that pursues youth, aging has a great influence on the psychological and emotional anxiety and depression of the middle-aged, rather than limited to the elderly. Above all, the middle-age

*This thesis is a revised version of Choi's master's thesis.

Received: February 13, 2021; $1^{\text {st }}$ Review Result: March 30, 2021; $2^{\text {nd }}$ Review Result: May 18, 2021

Accepted: June 30, 2021 
generations are more negative and experience anxiety about aging than actual elderly people[3]. For this reason, in exploring the relationship between aging anxiety and depression, which are of interest in this study, the researchers will compare the middle aged and the elderly generation.

In the previous studies on the relationship between aging anxiety, depression, and self-esteem, it was conducted focusing mainly on a single subject[4-6]. For example, These single subjects such as middleaged women[4], middle-aged men[4], elderly women[5], and elderly men[6], are focused on research on aging anxiety. Accordingly, there is insufficient information on whether aging anxiety is really different or similar between middle-aged and elderly generation, so this study is focused on academic interest in whether there are differences in aging anxiety, self-esteem, and depression between the two groups[5-6].

On the other hand, aging anxiety has a great influence on overall life, such as depression and selfesteem, as it continues from the middle-aged to the elderly[7]. Aging anxiety negatively affects developmental tasks such as life satisfaction, psychological well-being, retirement preparation, adaptation to aging such as successful aging, or entering a healthy old age. Negative images of aging and the conviction of stereotypes threaten the well-being of the elderly, and ultimately, the thought that one's own life cannot be controlled becomes widespread. In the end, aging is bound to become an object of fear and anxiety. As a result, the elderly may experience difficulties accompanied by depression. In addition, since aging anxiety affects self-esteem, and self-esteem affects depression, self-esteem among middle-aged and elderly generations is predicted to play a mediating role in the relationship between anxiety and depression[7].

Therefore, the purpose of this study is to verify the mediating effect of self-esteem in the relationship between aging anxiety and depression in the middle-aged and elderly generations. Specific research questions include: First, does aging anxiety of middle-aged and older generations affect depression? Second, does self-esteem have a mediating effect on the relationship between aging anxiety and depression in middle-aged and older generations?. The results of this study is intended to provide basic data for establishing measures to prevent or lessen depression by reducing aging anxiety and improving self-esteem in the middle-aged and elder generation.

\section{Theoretical Background}

In order to verify the mediating effect of self-esteem in the relationship between aging anxiety and depression, this section analyzed previous studies on the relationship between aging anxiety and depression, aging anxiety and self-esteem, and self-esteem and depression.

First, aging anxiety affects depression[8]. In the case of middle-aged people, the higher the aging anxiety, the higher the feeling of depression. The experience of aging due to deterioration of one's own physical abilities and health, and the burden of family and social roles experienced in the people can eventually lead to fear of aging and aging anxiety[9]. This is because the psychological instability of the middle-aged has an effect on reducing life satisfaction. Even in old age, aging anxiety affects depression. The elderly with high aging anxiety generally suffer from high levels of depression, and in old age, psychological and social functions diminish, resulting in a low sense of belonging, increasing anxiety caused by aging, which leads to high depression. This leads to extremes of suicidal thoughts. They also said that it is difficult to maintain a satisfactory life in general when excessive anxiety, psychological tension, and physical abnormalities such as headache, cold and insomnia appear[10].

Second, aging anxiety affects self-esteem[8]. As aging anxiety is a psychological factor that does not positively accept individual changes, it causes a sense of helplessness and negatively affects self-esteem. Persistent and serious aging anxiety in old age can have a negative effect on self-esteem, and aging anxiety in old age has a negative effect on social interactions, and subjective fear and anxiety about aging and old age can lead to greater disability. 
Third, self-esteem affects depression. People with high self-esteem cope well with negative events or emotions and are highly adaptable. On the other hand, people with low self-esteem tend to accept negative emotions such as depression as they are, rather than try to overcome negative events. According to the trait-stress model, depression tends to persist when self-esteem is low, and that interaction with stress affects depression. It was found that high stress, low self-esteem, and perception of low health status affect depression in women, and low self-esteem and high stress are factors that increase depression in men. In addition, people with low self-esteem think negatively about everything related to themselves because of the wrong cognitive system about themselves and their environment compared to those with high self-esteem and interpret anything related to themselves in the direction of blaming themselves. It has been reported to experience more severe depression from negative events[11].

Therefore, when these previous studies are put together, self-esteem is expected to play a mediating role in the effect of aging anxiety on depression, but no studies have been found that verified this process targeting the middle and old age.

\section{Method}

\subsection{Research Subjects and Data Collection Procedure}

The subjects of this study are middle-aged people aged 40 to 64 years old and elderly men and women living in communities over 65 years old. For data collection, a survey was conducted in December 2019 targeting middle-aged and elderly people living in Seoul, Gyeonggi, and Seosan. The survey was conducted with the cooperation of the head of the institution and the help of the person in charge by visiting the local lifelong learning center, general social welfare center, and volunteer center. Finally, 200 copies of middle aged and 286 copies of the elderly were used for the analysis. There are three variables used in this analysis, with 200 cases in middle age and 286 cases in old age, with no problem in the analysis.

\subsection{Instrument}

As the aging anxiety measurement, the Anxiety about Aging Scale (AAS)[12] was supplemented by Kim[13], and the scale used in the'Comparison Study of Aging Anxiety between Korean and American Old Men was used in this research. The AAS scale used in this study can be measured for all ages, and is also a scale that can be used in comparative studies involving multiple age groups. As a result of the reliability analysis, two questions that lowered the credibility (I am afraid that all my friends will die first in old age, I have never cheated my age to look younger) were deleted. For the reliability of the scale, the Cronbach's alpha value was .827 for the middle-aged and .711 for the elderly.

For depression, 10 items of depression revised by Choi[14] among SCL-90 were used. The higher the score, the higher the depression. It is a 5-point Likert scale, and the reliability of the scale, Cronbach's alpha value was .905 for the middle-aged and .860 for the elderly.

Self-esteem was translated and used by Rosenberg's[15] Self-esteem Scale. It is a 5-point Likert scale, and questions 3, 5, 8, 9, and 10 are reverse-coded, and the higher the score, the higher the self-esteem. The reliability of the scale, Cronbach's alpha was .843 for the middle-aged and .678 for the elderly.

\subsection{Characteristics of Study Subjects}

In order to find out the socio-demographic characteristics of the study subjects, frequency analysis was conducted by dividing into middle-aged and elderly generations. 
First, looking at the gender of the middle-aged generation, there are 131 women (65.5\%) and 69 men (34.5\%). The ages are evenly distributed, with 85 (42.5\%) being the most from 50 to 59 years old, 72 (36.0\%) from 40 to 49 years old, and $43(21.5 \%)$ from 60 to 64 years old. The average age is 52.49 years. In terms of marital status, 174 people $(87.0 \%)$ are married, most of them have spouses, and 26 $(13.0 \%)$ are divorced, bereaved, and so on. In terms of education, $114(57.0 \%)$ had college graduates or higher, and $73(36.5 \%)$ had middle and high school graduates.

Next, looking at the socio-demographic characteristics of the elderly, there are 144 (50.3\%) female seniors and $142(49.7 \%)$ male seniors. In terms of age, 138 people (48.3\%) are 70 to 79 years old, half of them, from 65 to 69 years old, 96 (33.6\%), and 52 (18.2\%) are those aged 80 and over. The average age is 73.29 years old. Looking at the marital status of the elderly, $204(71.3 \%)$ had a spouse, and 82 (28.7\%) of the elderly are either divorce, widowed, or unmarried. In terms of the education level of the elderly, 131 (46.8\%) middle and high school graduates and $120(42.9 \%)$ graduates from elementary school and below. The ratio of middle and high school graduates was similar, but there were $29(10.4 \%)$ highly educated people with a college degree or higher, which was low.

\subsection{Analysis}

Reliability analysis, t-test, and correlation analysis were performed using SPSS 21.0, and analysis was performed using model 4 of the SPSS PROCESS macro proposed by Hayes[16] to examine the mediating effect. The mediating effect (indirect effect) was suggested by Zhao et al.[17] and Rucker et al.[18].

\section{Results}

\subsection{The Difference in Variables between the Middle-aged and Elderly}

When looking at the differences in aging anxiety, depression, and self-esteem between the middleaged and elderly generations, as shown in [Table 1], all showed statistically significant differences. That is, aging anxiety and depression were higher in the elderly, and self-esteem was higher in the middleaged generation.

[Table 1] Align Differences in Aging Anxiety, Depression , and Self-esteem between Middle-aged and Eldrely Generations $(\mathrm{N}=486)$

\begin{tabular}{|c|c|c|c|c|c|}
\hline & & $\mathrm{N}$ & $\mathrm{M}$ & S.D & $\mathrm{t}$ value \\
\hline \multirow{2}{*}{ Aging anxiety } & Middle-aged & 200 & 2.778 & 0.471 & \multirow{2}{*}{$-4.107^{* * *}$} \\
\hline & Elderly & 286 & 2.944 & 0.385 & \\
\hline \multirow{2}{*}{ Depression } & Middle-aged & 200 & 2.224 & 0.636 & \multirow{2}{*}{$-6.040^{* * *}$} \\
\hline & Elderly & 286 & 2.579 & 0.639 & \\
\hline \multirow{2}{*}{ Self-esteem } & Middle-aged & 200 & 3.679 & 0.530 & \multirow{2}{*}{$7.776^{* * *}$} \\
\hline & Elderly & 286 & 3.329 & 0.419 & \\
\hline
\end{tabular}

In addition, when looking at the average of each variable, the middle-aged generation had 2.778 points out of 5 points for aging anxiety, which was lower than the median value, and the depression level was low at 2.224. However, self-esteem was 3.679 points higher than the median value. In the case of the elderly, the aging anxiety score was 2.943 out of 5 points, which was lower than the median value, and the depression sensitivity was also low at 2.579 . However, self-esteem was 3.329 points higher than the median value. Therefore, it can be seen that both middle-aged and elderly generations have low aging 
anxiety and depression, but high self-esteem.

Next, the correlation between aging anxiety, depression, and self-esteem in the middle-aged and elderly generations was analyzed. As shown in [Table 2], the correlation between all variables was significant. In both the middle-aged and older generations, the higher the aging anxiety, the higher the depression and the lower the self-esteem. Also, the higher the self-esteem, the lower the depression. When looking at the correlation coefficient, the middle-aged from .354 to .690, and the elderly from .237 to .539 , and therefore it is judged that there is no problem of multicollinearity.

[Table 2] Correlation and Technical Statistics

\begin{tabular}{|c|c|c|c|c|}
\hline & & Aging anxiety & Depression & Self-esteem \\
\hline \multirow{3}{*}{$\begin{array}{l}\text { Middle- } \\
\text { aged } \\
(\mathrm{N}=200)\end{array}$} & Aging anxiety & & & \\
\hline & Depression & $.458^{* * *}$ & & \\
\hline & Self-esteem & $-.354^{* * *}$ & $-.690^{* * *}$ & \\
\hline \multirow{3}{*}{$\begin{array}{l}\text { The elderly } \\
(\mathrm{N}=286)\end{array}$} & Aging anxiety & & & \\
\hline & Depression & $.438^{* * *}$ & & \\
\hline & Self-esteem & $-.237^{* * *}$ & $-.539^{* * *}$ & \\
\hline
\end{tabular}

\subsection{The Mediating Effect of Self-esteem in the Relationship between Aging Anxiety and Depression}

\subsubsection{The Mediating Effect of Self-esteem in the Relationship between Aging Anxiety and Depression in Middle-aged Generation}

Aging anxiety in the middle and old age had a positive effect on depression $(\beta=.618, p<.001)$. In other words, the higher the aging anxiety, the higher the depression ([Table 3], [Fig. 1]).

[Table 3] Effects of Aging Anxiety on Depression in Middle-aged Generation ( $N=200)$

\begin{tabular}{|c|c|c|c|c|c|c|}
\hline \multicolumn{7}{|c|}{ Direct effect model (dependent variable: depression) } \\
\hline Variable & Coeffect & SE & $\mathrm{t}$ value & $\mathrm{p}$ & LLCI & ULCI \\
\hline Constant & .507 & .240 & 2.110 & .000 & .033 & .980 \\
\hline Aging anxiety & .618 & .085 & 7.253 & .006 & .450 & .786 \\
\hline \multicolumn{7}{|c|}{$\mathrm{R}^{2}=.201, \mathrm{~F}=52.607, \mathrm{p}=.000$} \\
\hline
\end{tabular}

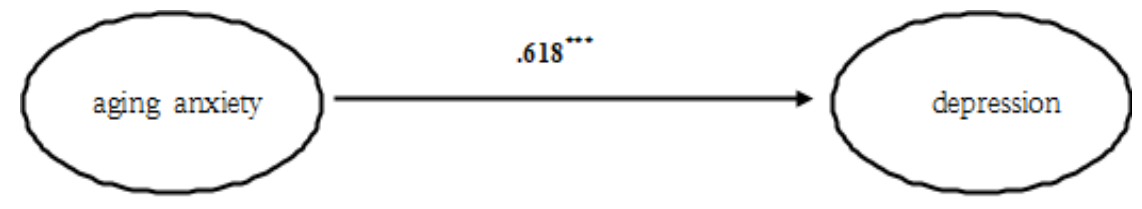

[Fig. 1] The Effect of Aging Anxiety on Depression in the Middle-aged Generation

Next, as a result of verifying the mediating effect of self-esteem in the relationship between aging anxiety and depression in the middle-aged generation([Table 4], [Fig. 2]), the mediating effects by route were all statistically significant.

Aging anxiety has a significant effect on self-esteem $(\beta=-.399, p<.001)$, self-esteem has a significant effect on depression $(\beta=-.720, p<.001)$, and self-esteem has a significant effect on aging anxiety. It can be seen that the relationship between aging anxiety and depression was mediated. In other words, the 
higher the aging anxiety, the lower the self-esteem, but the higher the self-esteem, the lower the depression, indicating that self-esteem mediates the relationship between aging anxiety and depression. Also, the effect of aging anxiety on depression was .618, but when self-esteem was added, it decreased to .330 , and a partial mediating effect was found in the relationship between aging anxiety and depression.

[Table 4] Mediating Effect of Self-esteem in the Relationship between Aging Anxiety and Depression in the Middle-aged Generation ( $\mathrm{N}=200)$

\begin{tabular}{|c|c|c|c|c|c|c|}
\hline Variable & Coeffect & $\mathrm{SE}$ & $\mathrm{t}$ value & $\mathrm{p}$ & LLCI & ULCI \\
\hline \multicolumn{7}{|c|}{ Parametric model (dependent variable: self-esteem) } \\
\hline Constant & 4.787 & .211 & 22.715 & .000 & 4.371 & 5.202 \\
\hline Aging anxiety & -.399 & .075 & -5.333 & .000 & -.546 & -.251 \\
\hline \multicolumn{7}{|c|}{ Parametric model (dependent variable: depression) } \\
\hline \multicolumn{7}{|c|}{$\mathrm{R}^{2}=.126, \mathrm{~F}=28.440, \mathrm{p}=.000$} \\
\hline Constant & 3.971 & .353 & 11.247 & .000 & 3.275 & 4.668 \\
\hline Aging anxiety & .330 & .071 & 4.668 & .000 & .190 & .469 \\
\hline Self esteem & -.724 & .063 & -11.542 & .000 & -.847 & -.600 \\
\hline \multicolumn{7}{|c|}{$\mathrm{R}^{2}=.529, \mathrm{~F}=110.482, \mathrm{p}=.000$} \\
\hline
\end{tabular}

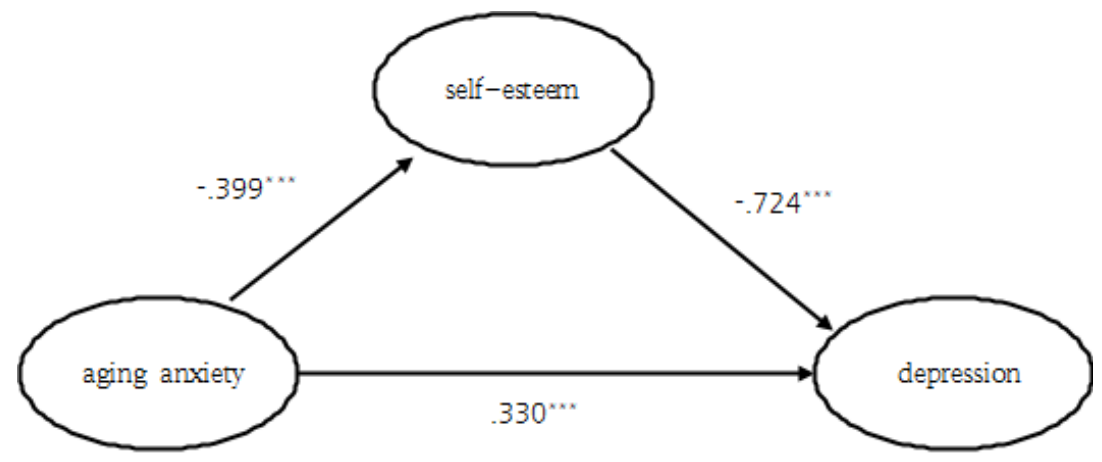

[Fig. 2] Mediating Effect of Self-esteem in the Relationship between Aging Anxiety and Depression in the Middle-aged Generation

However, since the significance of the indirect effect (mediated effect) is not known in [Table 3] and [Table 4], and [Fig. 1] and [Fig. 2], the indirect effect was verified according to the proposals[17][18]. As a result, in the path of aging anxiety $\rightarrow$ self-esteem $\rightarrow$ depression, the bootstrap value was .289 , and there was no ' 0 ' value between the upper and lower limits (.147 to .453), so the indirect effect of selfesteem was verified to be significant[Table 5].

[Table 5] Self-esteem Mediated Indirect Effect Verification in Middle-aged Generation ( $N=200)$

\begin{tabular}{|c|c|c|c|c|}
\hline & Effect & SE & LLCI & ULCI \\
\hline Indirect effect & .289 & .078 & .147 & .453 \\
\hline
\end{tabular}

\subsubsection{The Mediating Effect of Self-esteem in the Relationship between Aging Anxiety and Depression in the Elderly}

It was found that aging anxiety in the elderly had a positive effect on depression $(\beta=.726, p<.001)$. 
That is, it was found that the higher the aging anxiety, the higher the feeling of depression ([Table 6], [Fig. 3]).

[Table 6] Effects of Aging Anxiety on Depression in the Elderly ( $\mathrm{N}=286)$

\begin{tabular}{|c|c|c|c|c|c|c|}
\hline \multicolumn{7}{|c|}{ Direct Effect Model (Dependent : Depression) } \\
\hline Variable & Coeffect & SE & $\mathrm{t}$ value & $\mathrm{p}$ & LLCI & ULCI \\
\hline Constant & .442 & .262 & 1.683 & .094 & -.075 & .958 \\
\hline Aging anxiety & .726 & .088 & 8.213 & .000 & .552 & .900 \\
\hline \multicolumn{7}{|c|}{$\mathrm{R}^{2}=192, \mathrm{~F}=67.456, \mathrm{p}=.000$} \\
\hline
\end{tabular}

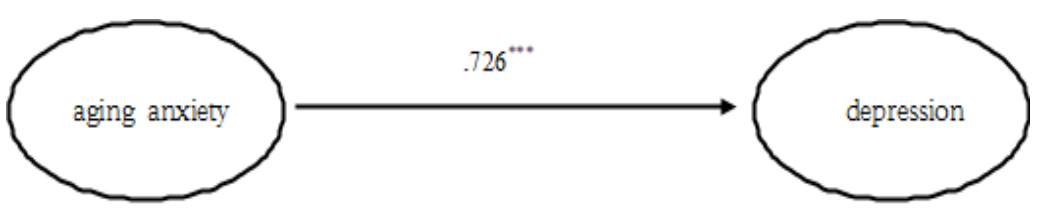

[Fig. 3] Effects of Aging Anxiety on Depression in the Elderly

[Table 7] Mediating Effect of Self-esteem in the Relationship between Aging anxiety and Depression in the Elderly $(\mathrm{N}=286)$

\begin{tabular}{|c|c|c|c|c|c|c|}
\hline variable & Coeffect & $\mathrm{SE}$ & $\mathrm{t}$ value & $\mathrm{p}$ & LLCI & ULCI \\
\hline \multicolumn{7}{|c|}{ Parametric model (dependent variable: self-esteem) } \\
\hline constant & 4.087 & .186 & 21.952 & .000 & 3.721 & 4.453 \\
\hline aging anxiety & -.257 & .063 & -4.105 & .000 & -.381 & -.134 \\
\hline \multicolumn{7}{|c|}{ Parametric model (dependent variable: depression) } \\
\hline \multicolumn{7}{|c|}{$\mathrm{R}^{2}=.056, \mathrm{~F}=16.851, \mathrm{p}=.000$} \\
\hline constant & 3.316 & .374 & 8.862 & .000 & 2.579 & 4.052 \\
\hline aging anxiety & .545 & .079 & 6.890 & .000 & .390 & .701 \\
\hline self esteem & -.703 & .073 & -9.685 & .000 & -.846 & -.560 \\
\hline \multicolumn{7}{|c|}{$\mathrm{R}^{2}=.393, \mathrm{~F}=91.644, \mathrm{p}=.000$} \\
\hline
\end{tabular}

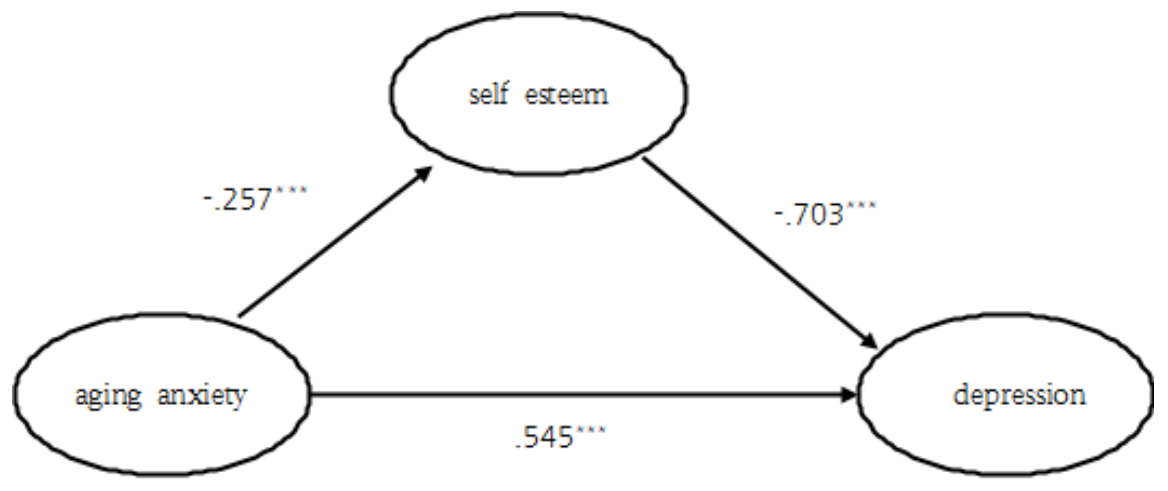

[Fig. 4] Mediating Effect of Self-esteem in the Relationship between Aging anxiety and Depression in the Elderly

Next, as a result of verifying the mediating effect of self-esteem in the relationship between aging anxiety and depression in the middle-aged generation [Table 7][Fig. 4], the mediating effects by route 
were all statistically significant. Aging anxiety has a significant effect on self-esteem $(\beta=-.257, p<.001)$, self-esteem has a significant effect on depression $(\beta=-.703, \mathrm{p}<.001)$, and self-esteem has a significant effect on aging anxiety. It can be seen that the relationship between depression and aging anxiety depression was mediated. That is, the higher the aging anxiety, the lower the self-esteem, but the higher the self-esteem, the lower the depression, indicating that self-esteem mediates the relationship between aging anxiety and depression. In addition, the effect of aging anxiety on depression was .726, but when self-esteem was added, it decreased to .545 , finding a partial mediating effect on the relationship between aging anxiety and depression.

However, since the significance of the indirect effect (mediated effect) is not known in [Table 6], [Table 7], [Fig. 3], and [Fig. 4], the indirect effect was verified according to the proposals of Zhao et al.[18] and Rucker et al.[19]. As a result, in the path of aging anxiety $\rightarrow$ self-esteem $\rightarrow$ depression, the bootstrap value was .181, and there was no ' 0 'value between the upper and lower limits (.076 .293), so the indirect effect of self-esteem was verified to be significant [Table 8].

[Table 8] Self-esteem Mediated Indirect Effect Verification in the Elderly $(\mathrm{N}=286)$

\begin{tabular}{|c|c|c|c|c|}
\hline & Effect & SE & LLCI & ULCI \\
Indirect effect & .181 & .055 & .076 & .293 \\
\hline
\end{tabular}

\section{Discussion and suggestions}

The purpose of this study was to determine the mediating effect of self-esteem in the relationship between aging anxiety and depression in the middle-aged and elderly generations living in local communities. Specifically, first, the differences in aging anxiety, self-esteem, and depression between the middle-aged and elderly generations were identified. Second, the mediating effect of self-esteem was investigated in the effects of aging anxiety on depression by middle-aged and elderly generations. To collect data on the middle-aged and the elderly, a survey was conducted in December 2019 targeting 300 middle-aged and elderly people living in Seoul, Gyeonggi, and Seosan. In the case of the middleaged, 200 copies were finally collected and used for analysis, and 286 copies were used for the elderly.

The main research results are summarized as follows.

First, aging anxiety and depression were higher in the elderly, and self-esteem was higher in the middle-aged generation. In the elderly, depression increases and self-esteem decreases due to anxiety and decrease in confidence due to various losses such as loss of health, loss of relationships, loss of decision-making power, and loss of respect from others[8]. As age is increased, the level of aging anxiety tends to increase[13]. However, this is different from a study showing that middle-aged anxiety is higher than that of the elderly and previous studies that show that there is no difference in the level of aging anxiety between age groups[12]. This is considered to be because the socio-demographic characteristics of the study subjects have diverse and complex correlations. Therefore, in a subsequent study, the difference in aging anxiety, self-esteem, and depression between the two groups needs to be verified using more objective and precise data.

In addition, looking at self-esteem, the middle-aged generation had higher self-esteem than the older generation. The middle-aged generation is more active in social activities and enjoys more benefits of education and culture than the elderly, and since it plays an economically leading role in the family, selfesteem may be higher than that of the elderly[19]. In addition, people in their 30s to 40s who have the most social activities have the highest self-esteem. Self-esteem increases with time for the youth and middle-aged[19] but decreases after age 60[20].

Second, as a result of verifying the mediating effect of self-esteem in the relationship between aging 
anxiety and depression, in both the middle-aged and elderly generations, the higher the aging anxiety, the higher the feeling of depression, but the effect of aging anxiety on the depression decreased when self-esteem was added. That is, the mediating effect of self-esteem was verified in the relationship between aging anxiety and depression. These results suggest that people with high self-esteem in both the middle-aged and elderly generations naturally recognize the phenomenon of aging and prepare selfmeasures to minimize or avoid anxiety caused by aging, and it is in the same vein as the study that the depression does not increase even when experiencing failure due to the negative attribution style[11].

In the relationship between aging anxiety and depression, they value themselves and think of themselves as being of value. An element that values one's own evaluation and values rather than evaluation of others is the mediating effect of self-esteem. The results of this study showing that the effects of aging anxiety on depression were reduced by the mediating effect of self-esteem suggest that in order to reduce depression in the middle-aged and elderly generation, it is necessary to improve selfesteem rather than reduce aging anxiety.

This study is meaningful in that it revealed that aging anxiety affects not only the elderly but also the middle-aged, and that self-esteem plays a mediating role in this relationship. Nevertheless, it points out that there are some limitations as follows. First, in this study, data collection was limited to Seoul, Gyeonggi, and Seosan. Therefore, there is a limitation in generalizing the results of this study. In future studies, a more valid study design is needed for sampling. Second, in this study, the same measurement tool was used for comparison between the middle-aged and elderly generations. The same scale was used for comparative analysis between the two groups, thus, it is suggested to conduct future studies that use a scale suitable for the characteristics of each group.

\section{References}

[1] KOSIS Top 100 Indicators, KOSIS, https://kosis.kr/conts/nsportalStats/ nsportalStats_0101Body.jsp?menuId=all, Oct $25(2020)$

[2] Moon-sin Jung, Hye-jin Kwon, A Study on the Middle-Aged Women's Perception on Facial Aging, International Journal of Advanced Nursing Education and Research, (2016), Vol.1, No.1, pp.81-86, DOI:10.21742/ijaner.2016.1.14

[3] S. M. Lynch, Measurement and Prediction of Aging Anxiety, Research on Aging, (2000), Vol.22, No.5, pp.533-558, https://doi.org/10.1177/0164027500225004

[4] J. Y. Yoo, Differences in Anxiety about Aging between Middle-aged Men and Women, Hanyang University, Master's Thesis, (2018)

[5] E. J. Lee, M. H Sung, Impacts of Health Perception, Aging Anxiety and Perception of Successful Aging on Health Promotion Behavior of Late Middle-Aged Women, Korean Journal of Women Health Nursing, (2017), Vol.23, No.3, pp.181-190, DOI : 10.4069/kjwhn.2017.23.3.181

[6] I. Jang, Male Baby Boomer' Generativity and Anxiety of Aging, Hanseo University, Master's Thesis, (2012)

[7] A. J. Flint, S. L. Rifat, Relationship between Clinical Variables and Symptomatic Anxiety in Late-life Depression, The American Journal of Geriatric Psychiatry, (2002), Vol.10, No.3, pp.292-296, https://doi.org/10.1097/00019442200205000-00008

[8] Seon-Nyeo Choi, Marital Relationship and Preschooler's Effortful Control: Focusing on the Multiple Mediating Effects of Mother's Depression and Parenting Stress, International Journal of Child Warfare Promotion and Management, (2018), Vol.2, No.1, pp.27-34.

[9] Kyoung-Mi Yang, The Relationship between Hwabyung Symptoms and Quality of Life among Middle-Aged Women, International Journal on Consulting Psychology for Patients, (2019), Vol.3, No.1, pp.15-22.

[10] Youngmi Ahn, Juyoung Ha, Oonhee Yee, Demand Survey for Development of Geriatric Depression Prevention 
Program, International Journal of Elderly Welfare Promotion and Management, (2018), Vol.2, No.2, pp.7-12.

[11] N. H. Jo, C. H. Seong, Effects of Stress and Self-esteem on Depression in Middle-aged Women and Middle-aged Men, Journal of the Korea Convergence Society, (2016), Vol.7, No.6, pp.89-97, DOI : 10.15207/JKCS.2016.7.6.089

[12] K. P. Lasher, P. J. Faulkender, Measurement of aging anxiety: Development of anxiety about aging scale, International Journal of Aging \& Human Development, (1993), Vol.37, No.4, pp.247-259, https://doi.org/10.2190/1U69-9AU2V6LH-9Y1L

[13] W. Kim, A Comparative Study on Anxiety about Aging of Older Adults in Korea and the United States, Korean Journal of Gerontological Social Welfare, (2010), No.47, pp.71-94, DOI : 10.21194/kjgsw..47.201003.71

[14] H. K. Choi, The process of caregiving stress among caregivers for the elderly in Korea: Social support, coping, and psychological health, Cornell University, Doctoral Dissertation, (1992)

[15] M. Rosenberg, Rosenberg self-esteem scale (RSE), Acceptance and commitment therapy, Measures package, (1965), Vol.61, No.52, p.18.

[16] A. F. Hayes, Partial, conditional, and moderated mediation: Quantification, inference, and interpretation, Communication Monographs, (2018), Vol.85, No.1, pp.4-40, https://doi.org/10.1080/03637751.2017.1352100

[17] X. S. Zhao, J. G. Lynch, Q. M. Chen, Reconsidering Baron and Keny: Myths and Truths about Mediation Analysis, Journal of Consumer Research, (2010), Vol.37, No.2, pp.197-206, DOI:10.1086/651257

[18] D. D. Rucker, K. J. Preacher, Z. L. Tormala, R. E. Pety, Mediation Analysis in Social Psychology: Current Practices and New Recommendations, Social \& Personality Psychology Compass, (2011), Vol.5, No.6, pp.359-371, https://doi.org/10.1111/j.1751-9004.2011.00355.x

[19] H. M. Kim, H. J. Moon, H. L. Chang, Self-esteem Changes among the Adults Across the Lifespan: Examining the Predictors of the Change, Korean Journal of Social Welfare, (2015), Vol.67, No.1, pp.83-107, DOI : 10.20970/kasw.2015.67.1.004

[20] U. Orth, K. H. Trzesniewski, R. W. Robins, Self-Esteem Development from Young Adulthood to Old Age: A CohortSequential Longitudinal Study, Journal of Personality and Social Psychology, (2010), Vol.98, No.4, pp.645-658, https://doi.org/10.1037/a0018769 